\title{
A Potent and Selective Dual Inhibitor of AXL and MERTK Possesses Both Immunomodulatory and Tumor-Targeted Activity
}

\author{
Jonathan Rios-Doria*, Margaret Favata, Kerri Lasky, Patricia Feldman, Yvonne Lo, \\ Gengjie Yang, Christina Stevens, Xiaoming Wen, Sarita Sehra, Kamna Katiyar, Ke Liu, \\ Richard Wynn, Jennifer J. Harris, Min Ye, Susan Spitz, Xiaozhao Wang, Chunhong He, \\ Yun-Long Li, Wenqing Yao, Maryanne Covington, Peggy Scherle and Holly Koblish \\ Incyte Research Institute, Wilmington, DE, United States
}

OPEN ACCESS

Edited by:

Jai Prakash

University of Twente, Netherlands

Reviewed by:

Marco A. Velasco-Velazquez, National Autonomous University of

Mexico, Mexico Mohamed Rahmani, University of Sharjah, United Arab Emirates

*Correspondence: Jonathan Rios-Doria jdoria@incyte.com

Specialty section: This article was submitted to

Cancer Molecular Targets and Therapeutics, a section of the journal Frontiers in Oncology

Received: 24 August 2020 Accepted: 09 November 2020 Published: 07 December 2020

Citation:

Rios-Doria J, Favata M, Lasky K, Feldman $P$, Lo $Y$, Yang G, Stevens $C$,

Wen X, Sehra S, Katiyar K, Liu K, Wynn R, Harris JJ, Ye M, Spitz S,

Wang $X$, He C, Li Y-L, Yao W, Covington $M$, Scherle $P$ and Koblish $H$ (2020) A Potent and Selective Dual Inhibitor of $A X L$ and MERTK

Possesses Both Immunomodulatory and Tumor-Targeted Activity.

Front. Oncol. 10:598477. doi: 10.3389/fonc.2020.598477
TYRO3, AXL, and MERTK constitute the TAM family of receptor tyrosine kinases, which play important roles in tumor growth, survival, cell adhesion, as well as innate immunity, phagocytosis, and immune-suppressive activity. Therefore, targeting both AXL and MERTK kinases may directly impact tumor growth and relieve immunosuppression. We describe here the discovery of INCB081776, a potent and selective dual inhibitor of AXL and MERTK that is currently in phase 1 clinical trials. In cellular assays, INCB081776 effectively blocked autophosphorylation of $\mathrm{XXL}$ or MERTK with low nanomolar half maximal inhibitory concentration values in tumor cells and Ba/F3 cells transfected with constitutively active AXL or MERTK. INCB081776 inhibited activation of MERTK in primary human macrophages and partially reversed M2 macrophage-mediated suppression of T-cell proliferation, which was associated with increased interferon- $\gamma$ production. In vivo, the antitumor activity of INCB081776 was enhanced in combination with checkpoint blockade in syngeneic models, and resulted in increased proliferation of intratumoral $\mathrm{CD} 4^{+}$and $\mathrm{CD} 8^{+} \mathrm{T}$ cells. Finally, antitumor activity of INCB081776 was observed in a subset of sarcoma patient-derived xenograft models, which was linked with inhibition of phospho-AKT. These data support the potential therapeutic utility of INCB081776 as an immunotherapeutic agent capable of both enhancing tumor immune surveillance and blocking tumor cell survival mechanisms.

Keywords: AXL, MERTK, PD-L1, immunotherapy, dual inhibitor, antitumor activity, small molecule

\section{INTRODUCTION}

The TAM kinases (TYRO3, AXL, and MERTK) have emerged as potential anticancer targets given their roles in regulating innate immune responses as well as driving tumor growth and proliferation (1-4). Growth arrest specific 6 (GAS6) and Protein S are the major and most extensively characterized ligands for these kinases $(2,5)$. Both GAS6 and Protein S are $\gamma$-carboxylated

Abbreviations: BID, twice a day; CFSE, carboxyfluorescein succinimidyl ester; DMSO, dimethyl sulfoxide; FBS, fetal bovine serum; GAS6, growth arrest specific 6; IC50, half maximal inhibitory concentration; IFN, interferon; PBMC, peripheral blood mononuclear cell; PBS, phosphate-buffered saline; PD-L1, programmed death ligand; PDX, patient-derived xenograft; SOCS, suppressor of cytokine signaling; TAM, TYRO3, AXL, and MERTK; Teff, effector T cell. 
proteins that bind to phosphatidylserine residues on the surface of apoptotic cells through their amino terminal Gla domains, whereas the carboxy domains of GAS6 and Protein S bind to the extracellular domains of the TAM kinases. Thus, GAS6 and Protein S function as bridge proteins for apoptotic cells and the TAM kinases, resulting in efferocytosis. This function of the TAM kinases to mediate apoptotic cell clearance acts to inhibit immunity. The process of efferocytosis mediated by TAMs maintains macrophages in an M2 polarized state and reduces expression of cytokines induced by M1-like macrophages (6). Further, this process clears antigens that may be exposed during cell death, and this may limit the immune response to neoantigens (5). AXL expressed on dendritic cells has also been shown to inhibit immune cell activity. AXL functions together with the type I interferon receptor to increase suppressor of cytokine signaling 1 (SOCS1) and SOCS3 expression, resulting in the termination of inflammatory tolllike receptor signaling (7). PROS1 expression is greatly increased in activated T cells, and PROS1 activates both AXL and MERTK on dendritic cells to attenuate T-cell activation (1). Loss of any member of the TAM family leads to autoimmunity in a gene dosage-dependent manner, consistent with the concept that TAM activation dampens the immune response $(2,3,8,9)$.

The TAM kinases also play a well-studied role in maintaining tumor cell growth, proliferation, and survival. In tumor cells, these kinases induce downstream signaling pathways including PI3K-AKT, RAF-MAPK, and PLC-PKC $(10,11)$. In this regard, activation of AXL contributes to several fundamental mechanisms of malignancy by promoting cancer cell migration and invasion, enhancing tumor angiogenesis, and facilitating cancer cell survival and tumor growth (11-13). Both AXL and MERTK overexpression and activation are critical mediators of acquired resistance to both conventional chemotherapies and targeted therapies (13). In many cancer types, overexpression and activation of AXL is generally correlated with malignant progression and poor prognosis $(12,14)$. AXL has been identified as a marker for tumors that have undergone epithelialmesenchymal transition, and inhibition of AXL has been shown to reverse epithelial-mesenchymal transition $(15,16)$. MERTK is amplified in a small percentage of esophageal carcinomas and is overexpressed in the absence of gene amplification in many cancers, including multiple myeloma, gastric, prostate, breast, melanoma, and rhabdomyosarcoma (5, 14). Silencing of the MERTK gene increased apoptosis, decreased colony formation, increased chemo-sensitivity, and decreased tumor growth in human non-small cell lung cancer models (11).

Based on the clear roles of AXL and MERTK in both immunosuppression and tumor-promoting mechanisms, we set out to develop a potent and selective small molecule dual inhibitor of both AXL and MERTK. Here we demonstrate that INCB081776, currently in phase 1 clinical trials, is a highly selective inhibitor of AXL and MERTK (17) and functions to inhibit AXL and MERTK through immunomodulatory as well as tumor-directed signaling mechanisms. We demonstrate that INCB081776 cooperates with checkpoint blockade to increase functional cytokine production as well as antitumor efficacy in syngeneic models. Lastly, we demonstrate antitumor activity of INCB081776 in sarcoma patient-derived xenograft (PDX) models.

\section{MATERIALS AND METHODS}

\section{Cell lines and Reagents}

H1299 cells (RRID: CVCL_0060) were maintained in RPMI1640 (RPMI, ThermoFisher Scientific, Carlsbad, CA, USA; \#11875-093) culture medium with 10\% fetal bovine serum (FBS; GE Healthcare, Chicago, IL, USA; \#SH30071.03) and obtained from American Type Culture Collection (\#CRL5803). Ba/F3 cells were obtained from German Collection of Microorganisms and Cell Cultures (DSMZ; Braunschweig, Germany; \#ACC 300) and grown in RPMI supplemented with $10 \%$ FBS plus $4 \mathrm{ng} / \mathrm{ml}$ interleukin-3. G361 cells (RRID: CVCL_1220) were obtained from American Type Culture Collection (\#CRL-1424) and maintained in RPMI medium containing $10 \%$ FBS. All human cell lines have been authenticated using short tandem repeat profiling within the last 3 years. All experiments were performed with mycoplasmafree cells. INCB081776 was produced by Incyte Corporation (ClinicalTrials.gov identifier \#NCT03522142). Human peripheral blood mononuclear cells (PBMCs) were obtained from normal leukapheresis of two healthy donors (Biological Specialties, Colmar, PA, USA).

\section{Biochemical Enzyme Assays, ATP Competition Assay, and Kinase Profile Assays}

Details of standard biochemical enzyme, ATP competition, and kinase profile assays conducted in our study are provided in the Supplementary Methods.

\section{Cell Proliferation Assays}

The cytoplasmic domain of AXL, MERTK, or TYRO3 fused with dimerization sequence and HA tag was cloned into a pMSCV (murine stem cell virus) vector with a puromycin-resistance marker to generate three constructs individually by electroporation into $\mathrm{Ba} /$ F3 cells. Single clones that were interleukin-3-independent and puromycin-resistant were selected and characterized. To evaluate effects on $\mathrm{Ba} / \mathrm{F} 3$ cell proliferation, 1,000 cells/well of $\mathrm{Ba} / \mathrm{F} 3, \mathrm{Ba} / \mathrm{F} 3$ AXL, Ba/F3-MERTK, or Ba/F3-TYRO3 cells were treated in the presence or absence of INCB081776 at various concentrations (10 concentration points with a three-fold dilution factor from the highest concentration of $10 \mu \mathrm{M}$ ) diluted in RPMI with $2 \%$ FBS for $48 \mathrm{~h}$ in a 384-well plate. Cell viability was measured by ATP assay (CellTiter-Glo Assay, Promega, Madison, WI, USA) according to the manufacturer's procedure. The data were converted to percent inhibition relative to dimethyl sulfoxide ( $0.4 \% \mathrm{DMSO})$ control, and half maximal inhibitory concentration $\left(\mathrm{IC}_{50}\right)$ curves were fitted using GraphPad Prism software (San Diego, CA, USA). 


\section{pAXL Inhibition Assay in H1299 Cells}

To measure the effect of INCB081776 on pAXL, H1299 cells were plated (30,000 cells/well) in 96-well tissue-culture plates (Costar, Corning Incorporated, Corning, NY, USA) and incubated overnight at $37^{\circ} \mathrm{C}$ with $5 \% \mathrm{CO}_{2}$. INCB081776 at an appropriate concentration was added and incubated for $1 \mathrm{~h}$ at $37^{\circ} \mathrm{C}$ with $5 \%$ $\mathrm{CO}_{2}$. rhGAS6 (R\&D Systems, Minneapolis, MN, USA; \#885-GSB) was added at $1 \mu \mathrm{g} / \mathrm{ml}$ to each well, and plates were incubated at $37^{\circ} \mathrm{C}$ with $5 \% \mathrm{CO}_{2}$ for $15 \mathrm{~min}$. Cells were harvested and lysed in $110 \mu \mathrm{l}$ of ice-cold lysis buffer (Cell Signaling Technology, Danvers, MA, USA; \#9803) with protease and phosphatase inhibitors (ThermoFisher Scientific; \#78446) for $1 \mathrm{~h}$ on ice and stored at $-80^{\circ} \mathrm{C}$ for ELISA. ELISA plates were prepared by incubating Greiner lumitrac high-binding plates with $8 \mu \mathrm{g} / \mathrm{ml}$ of anti-AXL antibody (R\&D Systems; MAB154) overnight at room temperature. The plates were washed and blocked with phosphate-buffered saline (PBS) with $0.1 \%$ bovine serum albumin. Cell lysates were loaded onto ELISA plates and incubated $2 \mathrm{~h}$ at room temperature. The plates were washed and incubated with LANCE Eu-W1024 anti-phospho-tyrosine antibody (Perkin Elmer, Waltham, MA, USA; \#AD0067) in DELFIA assay buffer (Perkin Elmer; \#4002-0010) for $2 \mathrm{~h}$ at room temperature, washed, and DELFIA Enhancement Solution (Perkin Elmer; \#4001-0010) was added. The plates were gently shaken for $15 \mathrm{~min}$ at room temperature and read on the PheraStar (BMG Labtech, Ortenberg, Germany). The data were converted to percent inhibition relative to DMSO control, and INCB081776 $\mathrm{IC}_{50}$ determination was performed by fitting the curve of percent inhibition versus the $\log$ of the inhibitor concentration using GraphPad Prism.

\section{Phospho-MERTK Inhibition Assay in G361 Cells}

Newly thawed G361 cells were allowed to recover for three passages before use, and only cells within 20 passages after thawing were used in the assay. Cells were kept under nonconfluent conditions and used in log-phase growth. Two milliliters of $1 \times 10^{6}$ cells $/ \mathrm{ml}\left(2 \times 10^{6}\right.$ cells/well) G361 cells were added to six-well tissue-culture plates (Corning Incorporated; \#3961) for 2 days. At the time of the assay, $1 \mathrm{ml}$ of medium was added to each well. To determine the activity of INCB081776, a stock solution of 5 mM INCB081776 in DMSO was used to make three-fold serial dilution of DMSO working stocks that were further diluted in culture medium, and $100 \mu \mathrm{l}$ of the diluted compound was added to each well with final concentrations ranging from $0.2 \mathrm{nM}$ to $1 \mu \mathrm{M}$. For control wells in the absence of INCB081776, $100 \mu \mathrm{l}$ of $0.22 \%$ DMSO was added to maintain the final $0.02 \%$ DMSO concentration in every sample. The mixtures of cells and compound were incubated for $1 \mathrm{~h}$ at $37^{\circ} \mathrm{C}$ in a humidified incubator supplemented with $5 \% \mathrm{CO}_{2}$, then $10 \mu \mathrm{l}$ of $55.5 \mu \mathrm{g} / \mathrm{ml}$ of MERTK-activating antibody (R\&D Systems; \#MAB8912; final concentration equal to $500 \mathrm{ng} / \mathrm{ml}$ ) in PBS was added to each well, except the unstimulated sample, and incubated for $30 \mathrm{~min}$ at $37^{\circ} \mathrm{C}$ in a humidified incubator supplemented with $5 \% \mathrm{CO}_{2}$. After incubation, each well was washed twice with $2 \mathrm{ml}$ of cold PBS. Lysis buffer (120 $\mu$ l; Cell Signaling Technology; \#9803) containing 1 mM PMSF, Halt phosphatase inhibitors (1:100 dilution;
ThermoFisher Scientific; \#78426), and protease inhibitors (1:50 dilution; Calbiochem ${ }^{\circledR} \# 535140$; MilliporeSigma, Burlington, MA, USA) was added to each sample and incubated on ice for $30 \mathrm{~min}$. The cell extracts were transferred to a 96-well $\mathrm{V}$ bottom plate, centrifuged at $3000 \mathrm{rpm}$ for $10 \mathrm{~min}$ at $4^{\circ} \mathrm{C}$, and the extracts were stored at $80^{\circ} \mathrm{C}$ until analysis by ELISA for phospho-MERTK (pMer; R\&D Systems; \#DYC2579). The optical density of the plate was measured using a Molecular Devices SpectraMax Plus microplate reader (Molecular Devices, San Jose, CA, USA) at $450 \mathrm{~nm}$ with wavelength correction at $540 \mathrm{~nm}$. Absorbance of the standards was plotted versus the concentration to generate a standard curve using four-parameter algorithm curve-fitting software (SOFTmax PRO application, Molecular Devices). pMERTK concentrations for unknown samples were determined by extrapolation from the standard curve. $\mathrm{IC}_{50}$ values were calculated by GraphPad Prism 7.0 using a nonlinear regression sigmoidal dose-response curve with variable slope.

\section{Phagocytosis Assay}

Peripheral blood of healthy donors was procured from BioIVT (Westbury, NY, USA). A stock concentration of $5 \mathrm{mM}$ INCB081776 in DMSO was diluted in PBS to concentrations of $10 \mathrm{nM}$ and $100 \mathrm{nM}$. Blood was incubated for $19 \mathrm{~h}$ with INCB081776 at concentrations of $10 \mathrm{nM}$ and $100 \mathrm{nM}$ along with a DMSO control. On the next day, blood was diluted with an equal volume of PBS and 2\% FBS, and PBMCs were isolated using Lymphoprep and SepMate-50 tubes (STEMCELL Technologies, Vancouver, BC, Canada) in accordance with the manufacturer's recommendations. The cells were counted, washed once in filtered PBS, and then were re-suspended in serum-free $\mathrm{Na}^{+}$medium $(145 \mathrm{mM} \mathrm{NaCl}, 5 \mathrm{mM} \mathrm{KCl}, 10 \mathrm{mM}$ HEPES, $0.1 \%$ bovine serum albumin, $5 \mathrm{mM}$ D-glucose, $\mathrm{pH} 7.5$, at $\left.37^{\circ} \mathrm{C}\right)$. The cells were then stained with APC conjugated antiCD14 and BV786 conjugated anti-CD16 (BD Biosciences, San Jose, CA, USA) in $\mathrm{Na}^{+}$medium for $30 \mathrm{~min}$ at room temperature. After $30 \mathrm{~min}$, the cells were washed and re-suspended in $\mathrm{Na}^{+}$ medium. PBMCs $\left(2 \times 10^{6}\right.$ cells in a volume of $100 \mu \mathrm{l} \mathrm{Na}^{+}$ medium) pre-labeled with APC anti-CD14 and BV786 antiCD16 mAb were added to $900 \mu \mathrm{l}$ pre-heated $\left(37^{\circ} \mathrm{C}\right) \mathrm{Na}^{+}$ medium, vortexed, and flow cytometry acquisition was performed on BD LSRFortessa X-20 (BD Biosciences) for $20 \mathrm{~s}$. To measure the bead uptake, $1 \mu \mathrm{l}$ of Fluoresbrite Yellow-Green latex microspheres (Polysciences, Warrington, PA, USA) was added and the mixture vortexed prior to acquisition for $20 \mathrm{~s}$. After $20 \mathrm{~s}$, the tube was vortexed again and acquisition was performed for $20 \mathrm{~s}$. This process of mixing the cells followed by acquisition was repeated until 4 min elapsed. Cells were gated by forward and side scatter and by CD14 APC, CD16 BV786 fluorescence signals. The mean fluorescence intensity of the Yellow-Green latex microspheres in $\mathrm{CD} 14^{++} \mathrm{CD} 16^{+}$cells over successive 20 -s intervals was plotted against time.

\section{Inhibition of MERTK Activity in Primary Macrophages}

PBMCs were separated using Ficoll-Paque density gradient centrifugation and any remaining red blood cells were lysed 
using $1 \times$ RBC Lysis Buffer (Cell Signaling Technology) for 5 min at room temperature. The PBMCs were washed with PBS before being enriched for monocytes using CD14 microbeads positive selection separation following the manufacturer's protocol as specified (AutoMacs Pro, Miltenyi Biotec, Bergisch Gladbach, Germany). The $\mathrm{CD} 14^{+}$cells were initially seeded at $1.5 \times 10^{6}$ per well in six-well plates in RPMI $+10 \%$ heat-inactivated FBS and $10 \%$ AB human serum (Sigma-Aldrich Corp., St Louis, MO, USA), $100 \mathrm{U} / \mathrm{ml}$ penicillin $+100 \mu \mathrm{g} / \mathrm{ml}$ streptomycin (Corning), supplemented with $100 \mathrm{ng} / \mathrm{ml}$ macrophage colony-stimulating factor (R\&D Systems; \#216-MC) and were cultured at $37^{\circ} \mathrm{C}, 5 \%$ $\mathrm{CO}_{2}$ for 10 days. Fresh macrophage colony-stimulating factor was added to the media every 3 days until the macrophages had attached. In preparation for the assay, the media was removed and the cells were re-fed with fresh media without human serum. INCB081776 stocks were prepared at $1000 \times$ in $100 \%$ DMSO and diluted 67-fold first into media and then a further 15 -fold when added to the macrophages. The macrophages were treated with INCB081776 for $2 \mathrm{~h}$ at $37^{\circ} \mathrm{C}, 5 \% \mathrm{CO}_{2}$. Five $\mu \mathrm{g} / \mathrm{ml}$ anti-MERTK antibody MAB8912 (R\&D Systems) was added to the macrophages for an additional $30 \mathrm{~min}$, at which time the cells were washed with cold PBS. All PBS was carefully aspirated from the wells and the dry plates were frozen at $-20^{\circ} \mathrm{C}$.

\section{Western Blotting}

Macrophages were allowed to thaw on ice before being lysed with $250 \mu \mathrm{l} /$ well of $1 \times$ Lysis Buffer (Cell Signaling Technology; \#9803) and Halt protease and phosphatase inhibitors (ThermoFisher Scientific) for $1 \mathrm{~h}$ at $4^{\circ} \mathrm{C}$. The lysed cells were scraped and transferred to an Eppendorf vial on ice. The lysates were centrifuged at $12,700 \mathrm{rpm}$ for $15 \mathrm{~min}$ at $4^{\circ} \mathrm{C}$. H1299 and PDX tumors were weighed and homogenized with lysis buffer supplemented with protease and phosphatase inhibitor cocktails (Roche, Basel, Switzerland; \#11836170001). Tumors were lysed on ice for $30 \mathrm{~min}$, followed by centrifugation at 13,000 rpm for $10 \mathrm{~min}$. Protein lysates were quantified using BCA Protein Assay Kit (Pierce, ThermoFisher Scientific; \#23225). Lysates were transferred to a new tube together with $6 \times$ Laemmli SDS sample buffer (Alfa Aesar, Haverhill, MA, USA) and the samples were heated for $6 \mathrm{~min}$ at $95^{\circ} \mathrm{C}$. Approximately $50 \mu \mathrm{g}$ of protein sample was loaded per well using Novex ${ }^{\mathrm{TM}} 8 \%-$ $16 \%$ Tris-Glycine Mini Gels or $4 \%-12 \%$ Tris-Glycine Novex WedgeGels (Invitrogen, Carlsbad, CA, USA). The proteins were transferred to a nitrocellulose membrane using an iBlot (ThermoFisher Scientific) dry blotting system. The membranes were blocked with $0.5 \%$ nonfat dry milk in wash buffer $(100 \mathrm{mM}$ $\mathrm{NaCl}, 10 \mathrm{mM}$ Tris-HCl, $\mathrm{pH}$ 8.0, 0.1\% Tween 20) for $1 \mathrm{~h}$ at room temperature. The primary antibody used for pMERTK was from PhosphoSolutions (Aurora, CO, USA; \#p186-749). The remaining antibodies were obtained from Cell Signaling Technology: MERTK (\#4319), phosphor-AXL (\#5724), AXL, (\#4566), GAS6 (\#67202), phosphor-AKT (\#4060), AKT (\#9272), and $\beta$-Actin (\#4970). Primary antibodies were added at 1:500 and 1:1,000, respectively, in $0.5 \%$ milk/wash buffer and rocked overnight at $4^{\circ} \mathrm{C}$. The membranes were washed three times in wash buffer before incubation with the secondary antibody (anti-rabbit IgG1-HRP; Cell Signaling Technology) at
$1: 2500$ in $0.5 \%$ milk/wash buffer for $2 \mathrm{~h}$ at room temperature. The membranes were washed again before the bands were detected with SuperSignal West Dura Extended Duration Chemiluminescent substrate (ThermoFisher Scientific) and were visualized using the Fluorochem $M$ Digital Imager (Protein Simple, San Jose, CA, USA).

\section{Macrophage Suppression of T-Cell Proliferation Assay}

Human PBMCs were isolated from the peripheral blood of healthy donors by density gradient centrifugation on FicollHypaque (GE Healthcare, Chicago, IL, USA; \#17-1440-02) followed by purification with anti-CD14 Microbeads (Miltenyi Biotec; \#130-050-201). Isolated CD14 ${ }^{+}$monocytes/macrophages were incubated with $100 \mathrm{ng} / \mathrm{ml}$ macrophage colony-stimulating factor (R\&D Systems; \#216-MC) and $50 \mathrm{ng} / \mathrm{ml}$ TGFß1 (R\&D Systems; \#240-B) at $37^{\circ} \mathrm{C}$ for 6 days; $100 \mu \mathrm{l} /$ well of $\mathrm{CD} 14^{+}$ macrophages were seeded at $0.5 \times 10^{6}$ cells $/ \mathrm{ml}$ in a 96-well round-bottomed culture plate (Costar; \#3799) and treated with INCB081776 overnight at $37^{\circ} \mathrm{C}$. CD $4^{+} \mathrm{CD} 25^{-}$effector $\mathrm{T}\left(\mathrm{T}_{\text {eff }}\right)$ cells were isolated using the Dynabeads regulatory $\mathrm{CD} 4{ }^{+} \mathrm{CD} 25^{+}$ T-cell kit (Life Technologies, Carlsbad, CA, USA; \#11363D) and the $\mathrm{T}_{\text {eff }}$ cells were labeled with carboxyfluorescein succinimidyl ester (CFSE) using the CellTrace CFSE Cell Proliferation kit (ThermoFisher Scientific; \#C34554). Freshly CFSE-labeled $\mathrm{T}_{\text {eff }}$ cells were mixed with Dynabeads Human T-Activator CD3/ CD28 (ThermoFisher Scientific; \#11132D) at a ratio of 5:1, then $100 \mu \mathrm{l} /$ well of the T-cells/beads mixture at $1 \times 10^{6}$ cells $/ \mathrm{ml}$ were added to the INCB081776-treated $\mathrm{CD} 14^{+}$macrophages and continue to culture at $37^{\circ} \mathrm{C}$ for 5 days. $\mathrm{T}_{\text {eff }}$ cells were analyzed by a flow cytometer (BD LSRFortessa X-20, BD Biosciences) and the cell-free supernatant was tested in the Luminex assay (Millipore; \#HCYTOMAG-60K_38plex) to measure the concentrations of different cytokines and chemokines.

\section{In Vivo Efficacy Studies}

MBT-2 mouse bladder carcinoma cells were obtained from the Japanese Collection of Research Bioresources Cell Bank and were maintained in DMEM supplemented with 10\% FBS. MC38 cells were obtained from the National Cancer Institute. 4T1 cells were obtained from American Type Culture Collection and maintained in RPMI media supplemented with $10 \%$ FBS. For MBT-2 experiments, $5 \times 10^{5} \mathrm{MBT}-2$ cells were inoculated subcutaneously into the right hind flank of 6- to 8-week-old $\mathrm{C} 3 \mathrm{H}$ mice or athymic nude mice (Charles River Laboratories, Wilmington, MA, USA). For MC38 studies, 12-week-old female C57BL/6 mice were inoculated with brei of MC38 tumors (in vivo passage 6) into the right hind flank. For $4 \mathrm{~T} 1$ studies, $7.5 \times 10^{5} 4 \mathrm{~T} 1$ cells were inoculated subcutaneously into the right hind flank of 6- to 8-week-old female $\mathrm{BALB} / \mathrm{c}$ mice or athymic nude mice (Charles River Laboratories). Mice were randomized by tumor volume with $n=10-12$ per group using Study Director software (Studylog Systems, South San Francisco, CA, USA). INCB081776 was dissolved in $50 \mathrm{mM}$ citrate buffer (Alfa Aesar, Ward Hill, MA, USA; \#J63008AP) in 0.5\% methylcellulose (Sigma-Aldrich Corp.; \#M0430) and was dosed orally twice a day (BID) continuously from the start to the end of study. In the MC38 study, anti-programmed death ligand 1 
(PD-L1) (Bio X Cell, West Lebanon, NH, USA; BE0101) was dosed intraperitoneally at $15 \mathrm{mg} / \mathrm{kg}$ twice a week. The vehicle group and INCB081776 group were also administered rat IgG2b control antibody twice a week (BE0090, Bio X Cell) beginning at the start of treatment. In the $4 \mathrm{~T} 1$ study, a single dose of anti-PD-L1 was dosed intraperitoneally at $15 \mathrm{mg} / \mathrm{kg}$ at the start of the study. In the combination studies, the vehicle control group and mice receiving anti-PD-L1 were dosed with vehicle BID continuously until the end of study. Vehicle and INCB081776 were dosed orally BID. Mice were monitored for tumor growth and overt tolerability over the course of the experiment. PDX tumor models were conducted at Champions Oncology (Hackensack, NJ, USA). PDX tumors were implanted into 6- to 8-week-old female athymic nude mice. When tumor volumes were approximately $150-250 \mathrm{~mm}^{3}$, mice were randomized by tumor volume and were administered INCB081776 at $30 \mathrm{mg} / \mathrm{kg}$ BID by oral gavage. Tumor volume was calculated using the formula $\left(\mathrm{L} \times \mathrm{W}^{2}\right) / 2$, where $\mathrm{L}$ and $\mathrm{W}$ refer to the length and width dimensions, respectively. Tumor growth inhibition was calculated using the formula $\left(1-\left(\mathrm{V}_{\mathrm{T}} / \mathrm{V}_{\mathrm{C}}\right)\right) \times 100$, where $V_{T}$ is the tumor volume of the treatment group on the last day of treatment, and $\mathrm{V}_{\mathrm{C}}$ is the tumor volume of the control group on the last day of treatment. Two-way analysis of variance with Dunnett's multiple comparisons test was used to determine statistical differences between treatment groups (GraphPad Prism).

Mice were housed at 10-12 animals per cage, and were provided enrichment and exposed to 12-h light/dark cycles. The number of mice used per treatment group was based on historical experience with each tumor model. Mice whose tumor volumes exceeded limits (10\% of body weight) were humanely euthanized by $\mathrm{CO}_{2}$ inhalation. Animals were maintained in a barrier facility fully accredited by the Association for Assessment and Accreditation of Laboratory Animal Care, International. All of the procedures were conducted in accordance with the US Public Service Policy on Human Care and Use of Laboratory Animals and with Incyte Animal Care and Use Committee Guidelines.

\section{Pharmacokinetic Studies}

On the last day of the MBT-2 efficacy study in $\mathrm{C} 3 \mathrm{H}$ mice (day 18) or nude mice (day 14), plasma was collected at $1,2,4,8$, and $16 \mathrm{~h}$ (C3H mice) or 2,4 , and $16 \mathrm{~h}$ (nude mice) after the last oral administration of INCB081776. Samples were collected in EDTA-coated microtubes (Greiner Bio-one, Kremsmunster, Austria; 450475). Plasma concentrations of INCB081776 were determined with a calibration curve prepared in plasma. The assay range was $1-5,000 \mathrm{nM}$. Study sample aliquots $(25 \mu \mathrm{l})$ were deproteinized with vigorous mixing with six volumes of acetonitrile $(150 \mu \mathrm{l})$ containing INCB073305 as the internal standard. After centrifugation, $100 \mu \mathrm{l}$ of the supernatants were transferred to a 96-well plate containing $200 \mu \mathrm{l}$ of water, mixed well, and analyzed by liquid chromatography-tandem mass spectrometry. Chromatography was performed using $2-\mu \mathrm{l}$ injections of extracts with an ACE C18-AR, $(30 \times 2.1 \mathrm{~mm}, 3 \mu \mathrm{m}$, at $40^{\circ} \mathrm{C}$ ) column under gradient conditions at a flow rate of $0.75 \mathrm{ml} /$ min. $0.1 \%$ formic acid in water and $0.1 \%$ formic acid in acetonitrile were used for mobile phases $\mathrm{A}$ and $\mathrm{B}$, respectively. Liquid chromatography-tandem mass spectrometry analysis was performed using a Shimadzu Nexera UHPLC system coupled to the electrospray ionization source (in positive ion mode) of a Sciex $6500+$ triple quadrupole mass spectrometer. Peak areas for the multiple reaction monitoring transitions $636.3>121.0$ for INCB081776 and $695.3>636.1$ for the internal standard were used to calculate Analyte/internal standard peak area ratios, which were then used to create linear regression calibration equations with $1 / \mathrm{x}^{2}$ weighting.

\section{Immunophenotyping and Cytokine Quantitation}

At the end of study, tumors were collected $4 \mathrm{~h}$ after the last dose of INCB081776 and placed on ice. Tumor samples were cut into 2-mm pieces and transferred to Miltenyi C Tubes (Miltenyi Biotec; $\# 130$-096-334). Tumor dissociation was conducted according to the manufacturer's protocol (Miltenyi Biotec; \#130-096-730). The filter was rinsed with cold PBS and samples pelleted. Red blood cells were lysed in Pharm Lyse (BD; \#555899). Cells were washed with PBS and re-suspended in PBS with Live/Dead stain (LifeTech Scientific, Shenzhen, China; \#L34966) for $15 \mathrm{~min}$ at room temperature. Cells were then washed in PBS and re-suspended in stain buffer (BD; \#554657), and the following antibodies were added to the samples for 30 min at $4^{\circ} \mathrm{C}$ : CD3 (BD; \#553062), CD45 (BD; \#564279), CD8 (BD; \#560182), CD4 (BD; \#552775), CD11b (BD; \#557657), F4/80 (eBiosciences, San Diego, CA, USA; \#12-4801-82), MHC Class II (BD; \#562564), and CD206 (BioLegend, San Diego, CA, USA; \#141708). Cells were then washed, fixed, and permeabilized with fixation/permeabilization buffer (eBioscience 00-5523-00). Cells were re-suspended in permeabilization buffer (eBioscience; 008333). Ki-67 antibody (BioLegend; \#652413) was added to the samples for $1 \mathrm{~h}$ at room temperature. Cells were then washed and re-suspended in stain buffer (BD; \#554657) for acquisition. M1 macrophages were identified within the $\mathrm{CD} 45^{+}, \mathrm{CD} 11 \mathrm{~b}^{+} \mathrm{F} 4 / 80^{+}$ population as MHC Class $\mathrm{II}^{\mathrm{Hi}} / \mathrm{CD} 206^{\mathrm{Lo}}$ and M2 macrophages were identified as MHC Class $\mathrm{II}^{\mathrm{Lo}} / \mathrm{CD} 206^{\mathrm{Hi}}$. Data were acquired on a BD Fortessa and analyzed with FlowJo software. Levels of interferon (IFN)- $\gamma$ within tumors were quantitated with a multiplexed protein detection kit according to the manufacturer's protocol (MesoScale Diagnostics, Rockville, MD, USA). Statistical significance was determined by one-way analysis of variance with Dunnett's multiple comparisons test (GraphPad Prism).

\section{RESULTS}

The biochemical potency of INCB081776 to inhibit the enzymatic activity of TAM family members was investigated by timeresolved fluorescence energy transfer assays using recombinant phosphorylated forms of the kinase domains for AXL, MERTK, and TYRO3. The average $\mathrm{IC}_{50}$ values from multiple lots of INCB081776 against AXL, MERTK, and TYRO3 were $0.61 \pm$ $0.31 \mathrm{nM}(n=18), 3.17 \pm 1.97 \mathrm{nM}(n=25)$, and $101 \pm 27 \mathrm{nM}$ $(n=25)$, respectively, demonstrating approximately 30 -fold selectivity over TYRO3 (Table 1). INCB081776 was also evaluated at $200 \mathrm{nM}$ in a comprehensive kinase study, which included 179 kinases. INCB081776 was approximately 60-fold selective for AXL 
and MERTK compared with c-MET and did not inhibit any additional kinases (Supplementary Table 1). These results demonstrate that INCB081776 is a potent and highly selective inhibitor of AXL and MERTK. The mode of inhibition with respect to ATP concentration was evaluated using a MERTK assay. The $\mathrm{IC}_{50}$ values of INCB081776 for MERTK increased linearly with ATP concentration, indicating an ATP-competitive mode of inhibition (Supplementary Figure 1).

To evaluate the cellular potency and selectivity within the TAM receptor family, mouse $\mathrm{Ba} / \mathrm{F} 3$ cell lines with stable expression of AXL, MERTK, or TYRO3 were generated. Treatment of stable $\mathrm{Ba} /$ F3 transfectants with INCB081776 potently inhibited the proliferation of $\mathrm{Ba} / \mathrm{F} 3$ cells expressing either AXL or MERTK with concentration required for $50 \%$ growth inhibition values of $16 \pm 11 \mathrm{nM}(n=52)$ and $14 \pm 4.9 \mathrm{nM}(n=65)$, respectively, but weakly inhibited the growth of TYRO3-expressing $\mathrm{Ba} / \mathrm{F} 3$ cells $\left(\mathrm{IC}_{50}=498 \pm 161 \mathrm{nM} ; n=59\right)$ and was inactive against parental $\mathrm{Ba} / \mathrm{F} 3$ cells $\left(\mathrm{IC}_{50}>4000 \mathrm{nM}\right.$ ) (Table 2 ). These cellular data are consistent with the biochemical data and confirm that INCB081776 is a potent inhibitor of AXL and MERTK, and is $>30$-fold more selective for AXL and MERTK than TYRO3. In addition to engineered recombinant cell lines, the ability of INCB081776 to modulate AXL and MERTK activity was evaluated in tumor cell lines expressing high levels of endogenous AXL or MERTK. The non-small cell lung cancer cell line H1299 has been shown to exhibit markedly increased AXL protein expression (18). INCB081776 treatment of $\mathrm{H} 1299$ cells potently inhibited pAXL with an $\mathrm{IC}_{50}$ value of $1.8 \pm 0.63 \mathrm{nM}(N=19$, Figure 1A). Similarly, the potency of INCB081776 in blocking MERTK autophosphorylation was evaluated in G361 cells, a melanoma cell line expressing high level of MERTK (19). As shown in Figure 1B, INCB081776 effectively blocked MERTK phosphorylation induced by MAB8912 in G361 melanoma cells with an $\mathrm{IC}_{50}$ value of $6.5 \pm 3.1 \mathrm{nM}(N=4)$.

The TAM family of kinases play a key role in efferocytosis. To study the role of INCB081776 in inhibiting phagocytic uptake, a phagocytosis assay using primary human monocytes was employed (Supplementary Figure 2) (20). Analysis of AXL and

TABLE 1 | Biochemical potency of INCB081776 against AXL, MERTK, and TYRO3.

\begin{tabular}{lcc}
\hline Recombinant kinase & $\begin{array}{c}\text { Biochemical IC50, } \\
\text { Mean } \pm \text { SD (nM) }\end{array}$ & Number of replicates (n) \\
\hline AXL & $0.61 \pm 31$ & 18 \\
MERTK & $3.17 \pm 1.97$ & 25 \\
TYRO3 & $101 \pm 27$ & 25 \\
\hline
\end{tabular}

TABLE 2 | Cellular potency of INCB081776 against AXL, MERTK, and TYRO3.

\begin{tabular}{lcc}
\hline Ba/F3 cell line & $\begin{array}{c}\text { BAF3 growth inhibition } \mathbf{I C}_{\mathbf{5 0}}, \\
\text { mean } \mathbf{\pm} \text { SD (nM) }\end{array}$ & Number of replicates (n) \\
\hline AXL & $16 \pm 11$ & 52 \\
MERTK & $14 \pm 4.9$ & 65 \\
TYRO3 & $498 \pm 161$ & 59 \\
WT (no kinase) & $>4,000$ & 11 \\
\hline
\end{tabular}

$1 C_{50}$, concentration required for $50 \%$ growth inhibition; SD, standard deviation.
MERTK expression in intermediate (CD14++CD16+) and classical (CD14++CD16-) monocytes from healthy donors revealed that intermediate monocytes had higher expression of MERTK than classical monocytes, consistent with a previous report (21) (Supplementary Figure 3A). Both cell populations also lacked AXL expression (Supplementary Figure 3B) (21). INCB081776 reduced microsphere uptake in both intermediate and classical monocytes in a concentration-dependent manner (Figure $\mathbf{2 A}$ and Supplementary Figure 3C). These data demonstrate that INCB081776 reduced phagocytic ability of intermediate and classical monocytes, likely through inhibition of MERTK.

It is thought that MERTK plays a predominant role on the phagocytic ability of immunosuppressive macrophages $(6,22,23)$. To explore the effect of INCB081776 in inhibiting MERTK in primary human macrophages, a macrophage-inhibition assay was employed. MAB8912 was discovered to be a MERTK agonist antibody, and addition of this antibody to primary human macrophages led to MERTK phosphorylation (Figure 2B). Pretreatment of macrophages with INCB081776 prior to agonism with MAB8912 blocked the induction of phosphorylated MERTK in a concentration-dependent manner with an $\mathrm{IC}_{50}$ of $1.6 \pm 0.4 \mathrm{nM}$ (Figure 2B). These data suggest that INCB081776 can inhibit MERTK activity in primary human immune cells. Recent work on monocyte-derived macrophages suggests that these macrophages may exhibit immunosuppressive function and can act as suppressor cells that inhibit T-cell proliferation (24). To examine the functional activity of INCB081776, the effects on macrophage-mediated suppression of T-cell proliferation was evaluated. Figure 2C demonstrates that INCB081776 partially reversed macrophage-mediated suppression of T-cell proliferation. This was associated with an increase in IFN $-\gamma$ production, suggesting that IFN- $\gamma$ release was a consequence of the increased T-cell proliferation (Figure 2D).

To explore the activity of INCB081776 in vivo, pharmacodynamic studies were initially performed to determine dose levels required for target inhibition. For these studies, the modulation of phosphorylated AXL in the H1299 tumor model was chosen. H1299 tumor-bearing SCID mice were dosed with INCB081776 at dose levels of 3, 10, or $30 \mathrm{mg} / \mathrm{kg}$, and tumors were evaluated for pAXL and total AXL expression. The $3 \mathrm{mg} / \mathrm{kg}$ dose level appeared to partially inhibit pAXL, whereas full inhibition was observed at the 10 and $30 \mathrm{mg} / \mathrm{kg}$ dose levels (Supplementary Figure 4A). A time course study was then performed where H1299 tumors were collected at various timepoints after tumor-bearing mice were administered a single $30 \mathrm{mg} / \mathrm{kg}$ dose of INCB081776. Supplementary Figure 4B demonstrates that pAXL was fully inhibited up to $8 \mathrm{~h}$ post-dose; however levels rebounded at $16 \mathrm{~h}$. These data suggested that twice daily dosing was appropriate for dosing INCB081776 in antitumor efficacy studies (18).

To test potential immunomodulatory activity of INCB081776 in vivo, antitumor efficacy studies were performed in syngeneic tumor models, which utilize immunocompetent mice. INCB081776 induced dose-dependent efficacy in the MBT-2 and 4T1 tumor models (Figures 3A, B). INCB081776 was well tolerated and no changes in body weight were observed (Supplementary Figures 5A, B). INCB081776 was also dosed 



FIGURE 1 | Inhibition of PAXL and pMERTK in tumor cell lines. (A) H1299 cells were pretreated with INCB081776 for $1 \mathrm{~h}$, then stimulated with recombinant human GAS6 for 15 min. Cell lysates were quantified for pAXL by ELISA $(N=19)$. (B) G361 cells were pretreated with INCB081776 for $1 \mathrm{~h}$ followed by 30 min of incubation with a MERTK agonist antibody, MAB8912 $(N=4)$. Levels of pMERTK were quantified by ELISA. Half maximal inhibitory concentration $\left(I_{50}\right)$ values were determined by fitting the curve of percent inhibition versus the log of INCB081776 concentrations using sigmoidal dose response with variable slope in GraphPad Prism. SEM, standard error of the mean.
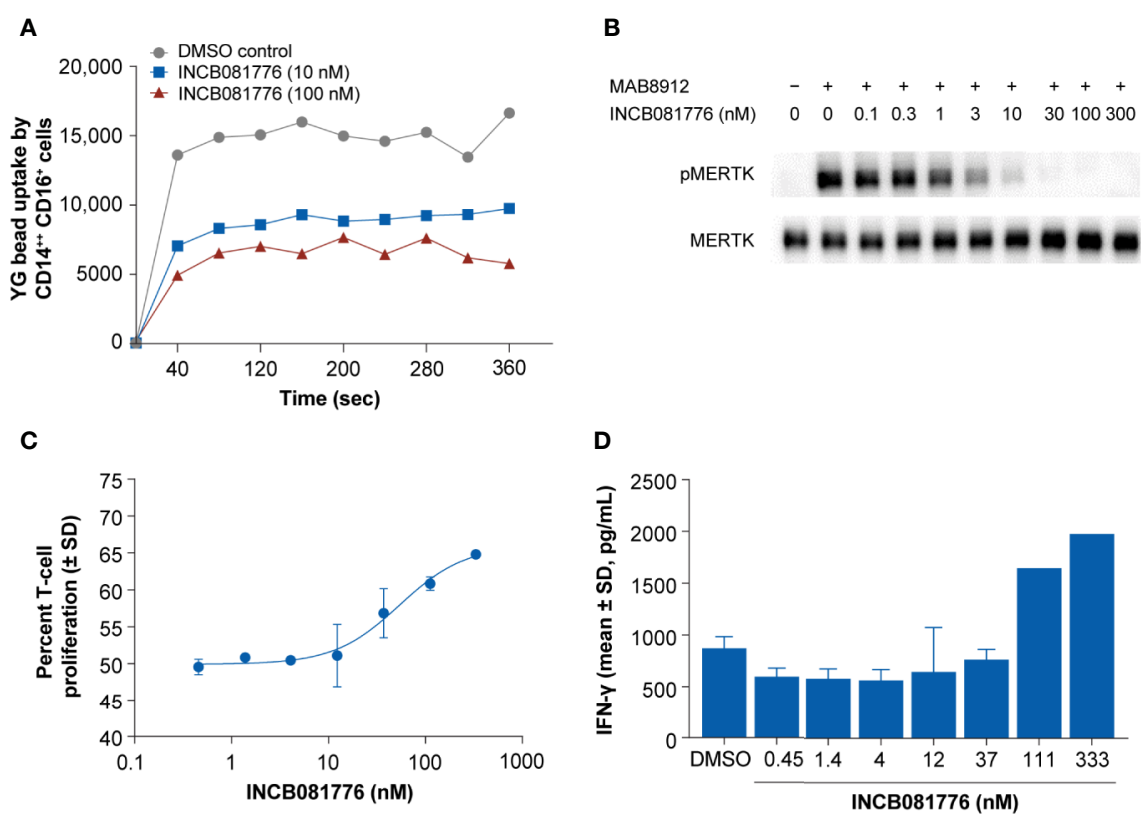

FIGURE 2 | Inhibition of phagocytosis and reversal of macrophage-mediated suppression of T cells by INCB081776 in primary human immune cells. (A) Human whole blood was treated with INCB081776 at $10 \mathrm{nM}$ and $100 \mathrm{nM}$ along with dimethyl sulfoxide control. Fluorescent microspheres were added to peripheral blood mononuclear cells (PBMCs) prelabeled with anti-CD14 and anti-CD16, and acquisition performed at intervals over a course of 4 min. Plot indicating the mean of the uptake of fluorescent microspheres by $\mathrm{CD} 14^{++} \mathrm{CD} 16^{+}$monocyte population over time is shown. Data are representative of three healthy donors. (B) Primary macrophages differentiated in vitro from human PBMCs were pretreated for $2 \mathrm{~h}$ with INCB081776 followed by stimulation with the MERTK-specific agonist antibody MAB8912 for an additional 30 min. Levels of phospho-MERTK (pMERTK) and total MERTK were determined by Western blot. (C) Primary macrophages differentiated in vitro from human PBMCs were pretreated with various concentrations of INCB081776 overnight, followed by incubation with carboxyfluorescein succinimidyl ester (CFSE)-labeled T cells for an additional 5 days. T-cell proliferation was determined by FACS analysis ( $N=2$ per group). (D) Following incubation of macrophages and T cells in the presence of increasing concentrations of INCB081776 as in panel (C), supernatants were collected and interferon (IFN)- $\gamma$ cytokine production was measured ( $N=2$ per group, except for 111 and $333 \mathrm{nM}[N=1])$. SD, standard deviation.

in nude mice, which lack functional T cells. There was no activity of INCB081776 in these mice, demonstrating the antitumor activity was dependent on $\mathrm{T}$ cells, at least in these models (Supplementary Figures 6A, B). In the MBT-2 antitumor efficacy studies, plasma was collected at various time points after the last oral dose of INCB081776. Pharmacokinetic analysis revealed dose-dependent plasma levels of INCB081776 in both $\mathrm{C} 3 \mathrm{H}$ and nude mice (Supplementary Figures 7A, 7B). 
In the MBT-2 model, INCB081776 treatment increased the ratio of M1-like to M2-like macrophages in a dose-dependent manner (Figure 3C). Given the functional reversal of macrophagemediated T-cell suppression observed in vitro, we hypothesized that INCB081776 might cooperate with checkpoint blockade to further boost antitumor responses. In the MC38 model, both INCB081776 and anti-PD-L1 had single-agent antitumor activity, but significantly higher activity in combination (Figure 4A). A similar enhanced combination effect was observed in the 4T1 model (Supplementary Figure 8). In the MC38 model, both single-agent treatments induced proliferation of $\mathrm{CD}^{+}$and $\mathrm{CD}^{+}$tumor-infiltrating lymphocytes, and to a higher degree in combination (Figure 4B). Combination treatment also resulted in increased IFN- $\gamma$ levels in tumors compared with single-agent treatment (Figure 4C). These data demonstrate that INCB081776 induced macrophage polarization, increased functional $\mathrm{CD}^{+}$and $\mathrm{CD}^{+}$T-cell activity, and combined with checkpoint blockade to enhance antitumor activity in vivo.

Besides playing roles in the regulation of immunity, AXL and MERTK provide growth and survival signals to the tumor cells directly $(15,25,26)$.Sarcomas are a mesenchymal tumor type where elevated AXL and MERTK expression has been observed
(25-27). Based on this, we evaluated INCB081776 for antitumor activity in sarcoma PDX models that expressed AXL and/or MERTK. INCB081776 induced a strong antitumor response in CTG-2041 with 94\% tumor growth inhibition (Figure 5A). A robust antitumor response was also observed in the CTG-1302 model (Figure 5B). In contrast, CTG-1339 was resistant to INCB081776 treatment (Figure 5C). Two additional sarcoma PDX models were partial responders and nine others were found to be nonresponsive to INCB081776 (Supplementary Table 2). To understand potential mechanisms responsible for the differences observed in responder compared with nonresponder models, we evaluated the impact of INCB081776 on AXL and MERTK activation as well as downstream signaling in CTG-2401 and CTG-1339 tumors (Figure 5D). Whereas INCB081776 inhibited pAXL and pMERTK in both models, CTG-2041 expressed significantly higher levels of total AXL and MERTK, especially pAXL and pMERTK (Figure 5D). These data imply that AXL and MERTK are strongly activated in CTG-2041. Interestingly, INCB081776 only inhibited pAKT in the CTG2041 model, which correlated with the antitumor efficacy. INCB081776 treatment also increased total MERTK levels in both tumor models, as well as GAS6 levels in CTG-2041. In total, these data suggest that INCB081776 can inhibit pAXL and
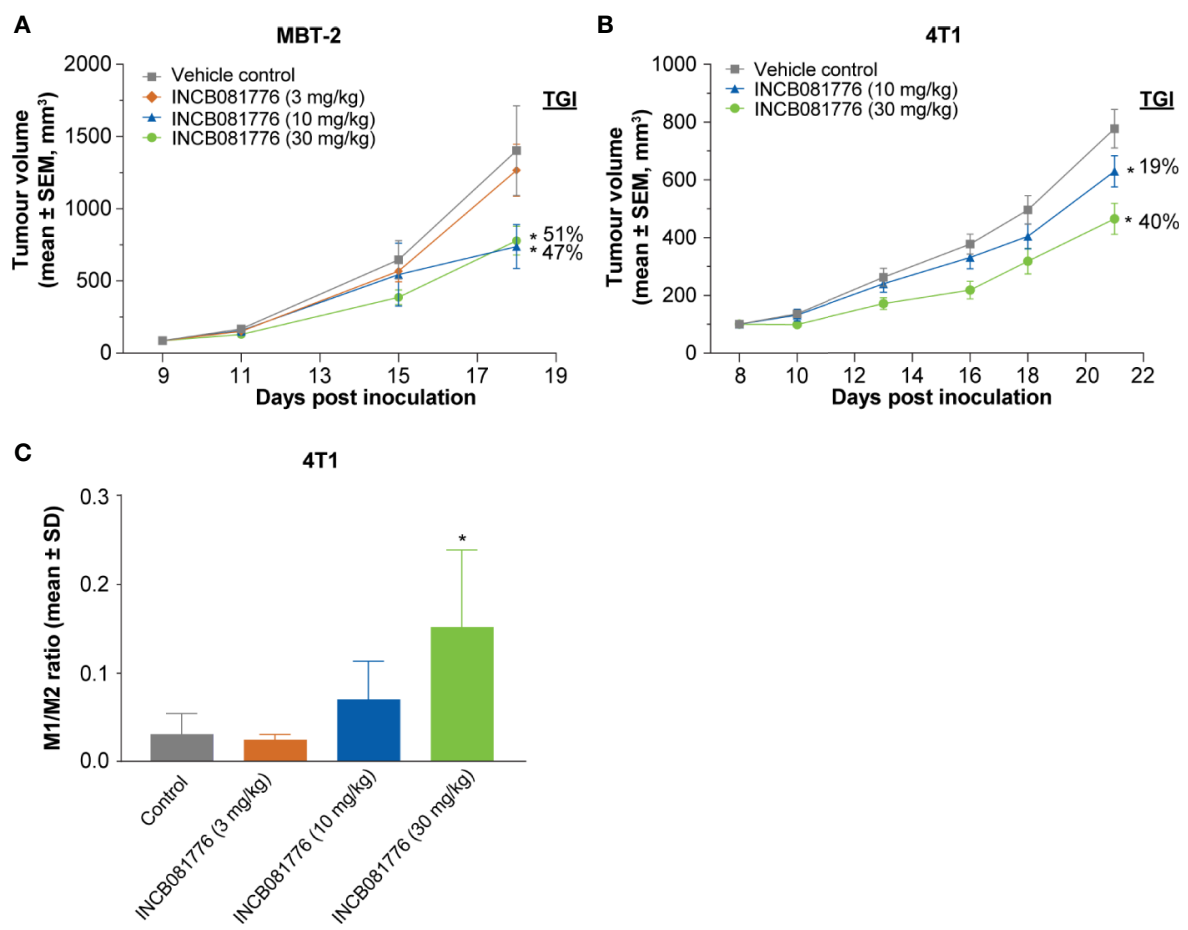

FIGURE 3 | Antitumor tumor activity of INCB081776 is dependent on the immune system in syngeneic models. (A) C3H mice bearing established MBT-2 tumors were administered INCB081776 orally twice a day at the indicated dose levels. $N=12$ mice per group. Tumor growth inhibition values are indicated. (B) BALB/C mice bearing established 4T1 tumors were dosed with INCB081776 at 10 and $30 \mathrm{mg} / \mathrm{kg}$ orally twice a day. $N=12$ per group. Statistical analysis was performed using two-way analysis of variance with Dunnett's multiple comparisons test in panels (A, B). ${ }^{\star} p<0.05$ compared with control. (C) Immunophenotyping of MBT-2 tumor-bearing mice treated with INCB081776 showed a dose-related increase in the M1-/M2-like macrophage ratio. $N=4$ per group. Statistical analysis was performed using one-way analysis of variance with Dunnett's multiple comparisons test ${ }^{*} p<0.05$ compared with control. SD, standard deviation; SEM, standard error of the mean; TGl, tumor growth inhibition. 
A

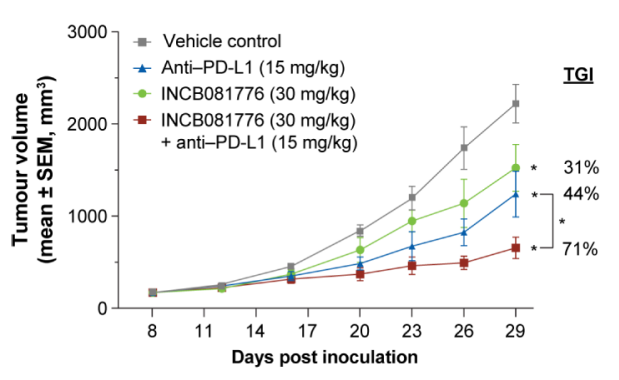

C

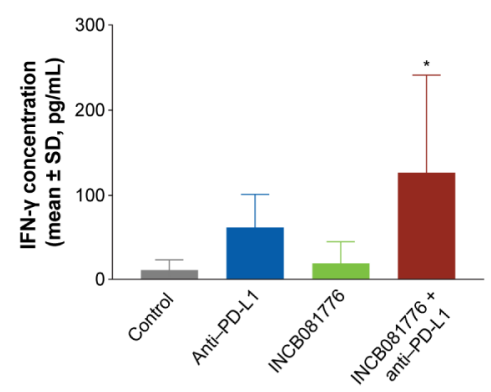

B

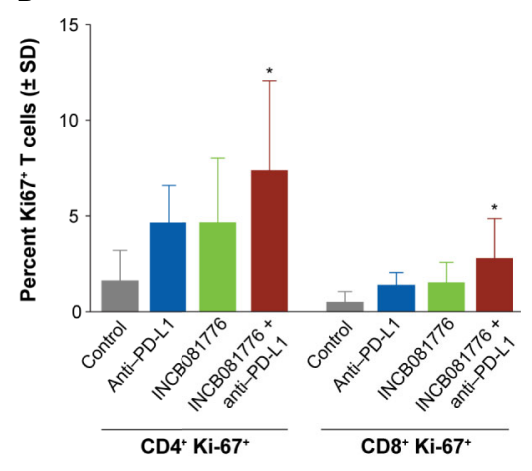

FIGURE 4 | INCB081776 displays enhanced antitumor activity in combination with checkpoint blockade. (A) C57BL/6 mice bearing established MC38 tumors were dosed orally twice a day with INCB081776, anti-programmed death ligand 1 (PD-L1) twice a week, or the combination. $N=12$ mice per group. Statistical analysis was performed using two-way analysis of variance with Dunnett's multiple comparisons test. ${ }^{*} p<0.05$ compared with control and for the between group comparison. (B) The percentage of $\mathrm{CD} 4^{+} \mathrm{Ki} i 67^{+}$and $\mathrm{CD} 8^{+} \mathrm{Ki} 67^{+}$cells were determined following treatment of MC38 tumor-bearing mice with INCB081776, anti-PDL1, or the combination. (C) Levels of interferon (IFN)- $\gamma$ in tumor extracts from mice at end of study were determined. $N=5$ per group in panels (B, C). Statistical analysis was performed using one-way analysis of variance with Dunnett's multiple comparisons test in panels $\mathbf{( B ,} \mathbf{C}) .{ }^{*} p<0.05$ compared with control. SD, standard deviation; SEM, standard error of the mean; TGl, tumor growth inhibition.

pMERTK in sarcoma PDX tumors and that pAKT inhibition correlated with antitumor efficacy.

\section{DISCUSSION}

AXL and MERTK kinases are increasingly being pursued as anticancer drug targets (28). The appeal of these kinases as anticancer targets stems from their roles in promoting tumor growth via both immunosuppressive and tumor-promoting mechanisms $(3,5,29)$. Based on these features, we developed a highly specific, dual inhibitor of AXL and MERTK. Selectivity data confirm that INCB081776 is selective and is equipotent for both AXL and MERTK. This dual targeting may be important in the design of small molecule inhibitors of the TAM family. It has been widely reported that AXL is a mediator of resistance to endothelial growth factor receptor inhibitors, as well as other targeted therapies and chemotherapies $(28,30,31)$. Recently, it was shown that MERTK is upregulated following genetic silencing or pharmacologic inhibition of AXL (32). Dual targeting of AXL and MERTK led to a more potent inactivation of downstream signaling and reduced tumor growth in vivo compared with targeting either kinase alone (32). These data highlight the possibility that a dual inhibitor of AXL and MERTK may circumvent resistance mechanisms that cells may develop to AXL inhibition. An additional report describing the pan-TAM inhibitor RXDX-106 concluded that inhibition of TYRO3 did not play a major role in the antitumor activity of the compound, supporting the idea that inhibition of AXL and MERTK are the most relevant targets in the TAM family (33).

It is believed that MERTK plays a predominant role in efferocytosis of M2-like macrophages, which produces immunosuppressive signals $(6,22,23)$. Studies have previously shown that simultaneously inhibiting efferocytosis by blocking TAMs and inhibiting indoleamine-2,3-dioxegenase 1 led to decreased immunosuppressive phenotypes and induced tumor regression (34). INCB081776 inhibited phagocytosis of human monocytes, consistent with anti-phagocytic properties of other MERTK inhibitors (35). In addition, INCB081776 effectively inhibited pMERTK upon activation of primary macrophages. Functionally, INCB081776 suppressed macrophage-mediated inhibition of $\mathrm{T}$-cell proliferation and induced IFN- $\gamma$ production, suggesting that MERTK inhibition plays a key role in this process. Therefore, inhibiting MERTK may play a role in reversing tumor-associated macrophages to an M1-like phenotype.

In vivo, INCB081776 possessed single-agent activity in the MBT2, MC38, and 4T1 models, and antitumor activity was enhanced in combination with a PD-L1-blocking antibody in the MC38 and 4T1 models. Consistent with in vitro findings, combination treatment in vivo led to an increase in $\mathrm{CD}^{+}$and $\mathrm{CD}^{+}$T-cell proliferation, which was associated with an increase in IFN- $\gamma$ production. Importantly, antitumor efficacy was lost when 

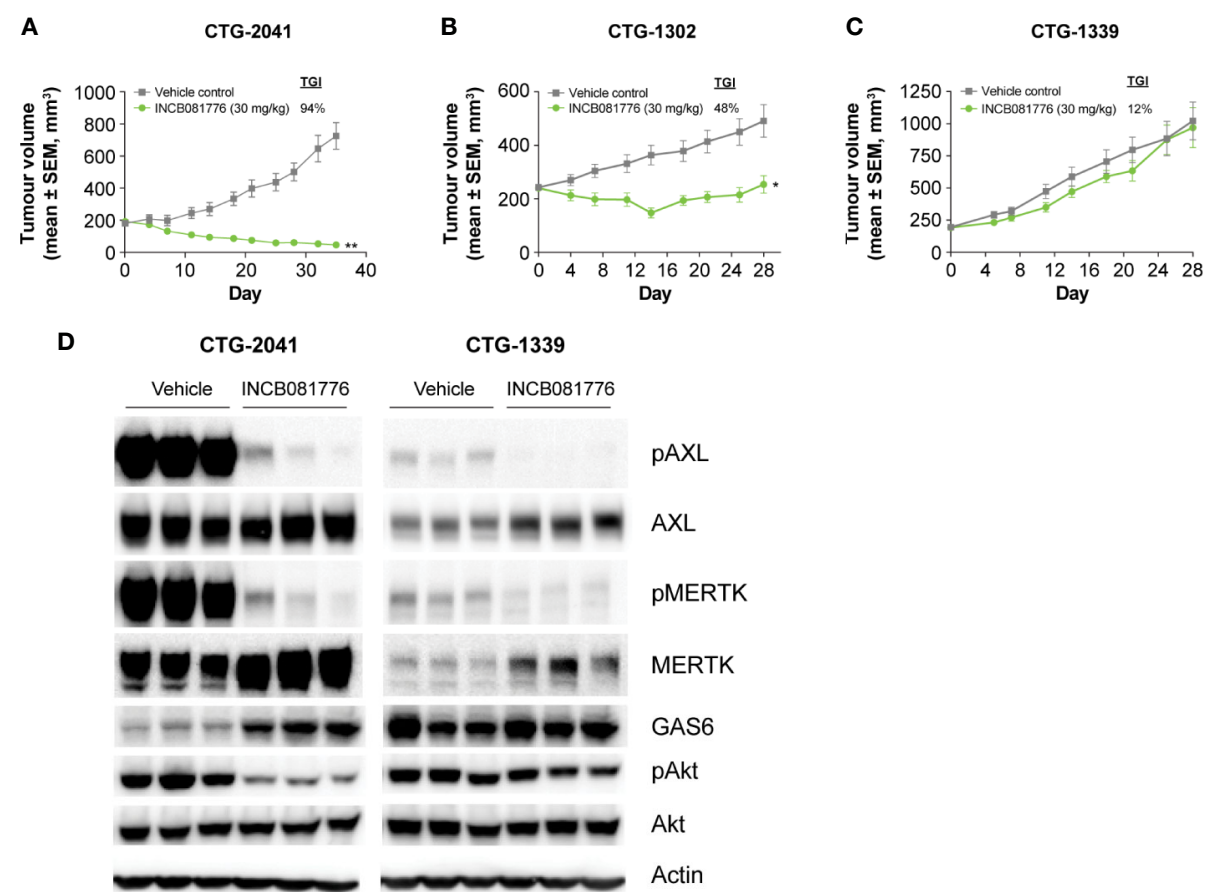

FIGURE 5 | Activity of INCB081776 in sarcoma PDX models. Athymic nude mice bearing (A) CTG-2041 (B) CTG-1302, or (C) CTG-1339 tumors were dosed orally twice a day (BID) with INCB081776 at $30 \mathrm{mg} / \mathrm{kg} . N=10$ mice per group in panels (A-C). Statistical analysis was performed using two-way analysis of variance with Sidak's multiple comparisons test (D) CTG-2041 or CTG-1339 tumors from mice treated with INCB081776 at $30 \mathrm{mg} / \mathrm{kg}$ twice a day (BID) or vehicle were lysed and processed by Western blot for pAXL, AXL, pMERTK, MERTK, GAS6, pAKT, AKT, and $\beta$-Actin. Three mice per group were analyzed. Western blot data were obtained from different gels. ${ }^{*} p<0.05 ;{ }^{* *} p<0.0001$; compared with control. SEM, standard error of the mean; TGl, tumor growth inhibition.

INCB081776 was dosed in immunodeficient animals bearing MBT2 and $4 \mathrm{~T} 1$ tumors, demonstrating that a functional immune system is required for efficacy in these syngeneic models. Overall, these data demonstrate a clear role of INCB081776 in modulating the immune system to promote antitumor responses.

Despite possessing antitumor activity that was dependent on the immune system, INB081776 also had direct antitumor activity in immunodeficient mice bearing sarcoma PDX tumors. A comparison between the nonresponsive CTG-1339 and responsive CTG-2041 sarcoma models demonstrated that while INCB081776 inhibited pAXL and pMERTK in both models, only pAKT was inhibited in CTG-2041. Our results are consistent with a prior report demonstrating that inhibition of pAKT is a biomarker of MERTK inhibition, although it is likely that inhibition of both pAXL and pMERTK contributed to the reduction of pAKT in the CTG-2041 model (36). These data indicate that AXL and MERTK are strong drivers of tumor growth in CTG-2041, and suggest that in the CTG-1339 model, there are other activated pathways that lead to AKT activation that are independent of AXL and MERTK. INCB081776 also modestly increased the levels of total MERTK. Although the reasons for this are not completely known, we speculate that INCB081776 may partially stabilize MERTK at the plasma membrane. The lack of response in other sarcoma PDX models tested may be due to different dependencies of these other tumors on other oncogenes and oncogenic pathways.
In summary, these data demonstrate that AXL/MERTK inhibition with INCB081776 hinders tumor growth by two distinct mechanisms-reversal of immunosuppression and ablation of intrinsic tumor cell survival signaling. We believe the profile of INCB081776 as a selective inhibitor of AXL and MERTK will provide an attractive potential treatment option for patients with cancer not only as a single agent, but also in rational combination with immunotherapeutic agents. A phase 1 clinical trial of INCB081776 is currently underway for patients with advanced solid tumors both as a single agent and in combination with PD-1 antibody INCMGA00012 (ClinicalTrials.gov identifier NCT03522142).

\section{DATA AVAILABILITY STATEMENT}

The original contributions presented in the study are included in the article/Supplementary Material, further inquiries can be directed to the corresponding author.

\section{ETHICS STATEMENT}

The animal study was reviewed and approved by Incyte's Animal Care and Use Committee. 


\section{AUTHOR CONTRIBUTIONS}

JR-D, RW, YL, JH, SSe, XWa, Y-LL, and HK designed experiments; MF, KLa, PF, GY, CS, XWe, SSe, KK, KLi, MY, and $\mathrm{CH}$ conducted experiments; JR-D drafted the manuscript and WY, SSp, MC, PS, and HK edited the manuscript; all authors reviewed and approved the manuscript for submission.

\section{FUNDING}

This study was funded by Incyte Corporation. The funder had the following involvement with the study: providing funding for laboratory materials to conduct these studies and salary support for authors.

\section{REFERENCES}

1. Carrera Silva EA, Chan PY, Joannas L, Errasti AE, Gagliani N, Bosurgi L, et al. $\mathrm{T}$ cell-derived protein $\mathrm{S}$ engages TAM receptor signaling in dendritic cells to control the magnitude of the immune response. Immunity (2013) 39:160-70. doi: 10.1016/j.immuni.2013.06.010

2. Lemke G, Rothlin CV. Immunobiology of the TAM receptors. Nat Rev Immunol (2008) 8:327-36. doi: 10.1038/nri2303

3. Paolino M, Penninger JM. The role of TAM family receptors in immune cell function: implications for cancer therapy. Cancers (2016) 8:E97. doi: 10.3390/ cancers 8100097

4. van der Meer JH, van der Poll T, van 't Veer C. TAM receptors, Gas6, and protein S: roles in inflammation and hemostasis. Blood (2014) 123:2460-9. doi: 10.1182/blood-2013-09-528752

5. Graham DK, DeRyckere D, Davies KD, Earp HS. The TAM family: phosphatidylserine sensing receptor tyrosine kinases gone awry in cancer. Nat Rev Cancer (2014) 14:769-85. doi: 10.1038/nrc3847

6. Myers KV, Amend SR, Pienta KJ. Targeting Tyro3, Axl and MerTK (TAM receptors): implications for macrophages in the tumor microenvironment. Mol Cancer (2019) 18:94. doi: 10.1186/s12943-019-1022-2

7. Rothlin CV, Ghosh S, Zuniga EI, Oldstone MB, Lemke G. TAM receptors are pleiotropic inhibitors of the innate immune response. Cell (2007) 131:112436. doi: 10.1016/j.cell.2007.10.034

8. Cohen PL, Caricchio R, Abraham V, Camenisch TD, Jennette JC, Roubey RA, et al. Delayed apoptotic cell clearance and lupus-like autoimmunity in mice lacking the c-mer membrane tyrosine kinase. J Exp Med (2002) 196:135-40. doi: 10.1084/jem.20012094

9. Lu Q, Lemke G. Homeostatic regulation of the immune system by receptor tyrosine kinases of the Tyro 3 family. Science (2001) 293:306-11. doi: 10.1126/ science. 1061663

10. Feneyrolles C, Spenlinhauer A, Guiet L, Fauvel B, Dayde-Cazals B, Warnault P, et al. Axl kinase as a key target for oncology: focus on small molecule inhibitors. Mol Cancer Ther (2014) 13:2141-8. doi: 10.1158/1535-7163.MCT-13-1083

11. Linger RM, Cohen RA, Cummings CT, Sather S, Migdall-Wilson J, Middleton $\mathrm{DH}$, et al. Mer or Axl receptor tyrosine kinase inhibition promotes apoptosis, blocks growth and enhances chemosensitivity of human non-small cell lung cancer. Oncogene (2013) 32:3420-31. doi: 10.1038/onc.2012.355

12. Graham DK, Dawson TL, Mullaney DL, Snodgrass HR, Earp HS. Cloning and mRNA expression analysis of a novel human protooncogene, c-mer. Cell Growth Differ (1994) 5:647-57.

13. Vouri M, Hafizi S. TAM Receptor Tyrosine Kinases in Cancer Drug Resistance. Cancer Res (2017) 77:2775-8. doi: 10.1158/0008-5472.CAN-162675

14. Linger RM, Keating AK, Earp HS, Graham DK. TAM receptor tyrosine kinases: biologic functions, signaling, and potential therapeutic targeting in human cancer. Adv Cancer Res (2008) 100:35-83. doi: 10.1016/S0065-230X (08)00002-X

\section{ACKNOWLEDGMENTS}

We thank Sunkyu Kim for critical review of the manuscript, Bobby Landman and $\mathrm{Yu} \mathrm{Li}$ for ADME (drug absorption, distribution, metabolism, and excretion) support, Yaoyu Chen, Hui Wang, Christine Gardiner, Mark Rupar, and Jun Li for technical support, and Elham Behshad, Zhenhai Gao, Bruce Ruggeri, and Mike Liu for thoughtful suggestions for the study. Editorial assistance was provided by Envision Pharma Group, Inc (Philadelphia, PA, USA).

\section{SUPPLEMENTARY MATERIAL}

The Supplementary Material for this article can be found online at: https://www.frontiersin.org/articles/10.3389/fonc.2020.598477/ full\#supplementary-material

15. Antony J, Huang RY. AXL-Driven EMT State as a Targetable Conduit in Cancer. Cancer Res (2017) 77:3725-32. doi: 10.1158/0008-5472.CAN-17-0392

16. Gjerdrum C, Tiron C, Høiby T, Stefansson I, Haugen H, Sandal T, et al. Axl is an essential epithelial-to-mesenchymal transition-induced regulator of breast cancer metastasis and patient survival. Proc Natl Acad Sci USA (2010) 107:1124-9. doi: 10.1073/pnas.0909333107

17. Favata M, Lasky K, Lo Y, Feldman P, Li J, Chen Y, et al. Abstract 3759: Characterization of INCB081776, a potent and selective dual AXL/MER kinase inhibitor. Proc Am Assoc Cancer Res Annu Meeting 2018 (2018) 78:3759-. doi: 10.1158/1538-7445.am2018-3759

18. Qu X, Liu J, Zhong X, Li X, Zhang Q. Role of AXL expression in non-small cell lung cancer. Oncol Lett (2016) 12:5085-91. doi: 10.3892/ol.2016.5356

19. Schlegel J, Sambade MJ, Sather S, Moschos SJ, Tan AC, Winges A, et al. MERTK receptor tyrosine kinase is a therapeutic target in melanoma. J Clin Invest (2013) 123:2257-67. doi: 10.1172/JCI67816

20. Gu BJ, Sun C, Fuller S, Skarratt KK, Petrou S, Wiley JS. A quantitative method for measuring innate phagocytosis by human monocytes using real-time flow cytometry. Cytometry A (2014) 85:313-21. doi: 10.1002/cyto.a.22400

21. Barth ND, Marwick JA, Heeb MJ, Gale AJ, Rossi AG, Dransfield I. Augmentation of Human Monocyte Responses to Lipopolysaccharide by the Protein S and Mer/Tyro3 Receptor Tyrosine Kinase Axis. J Immunol (2018) 201:2602-11. doi: 10.4049/jimmunol.1800249

22. Zizzo G, Hilliard BA, Monestier M, Cohen PL. Efficient clearance of early apoptotic cells by human macrophages requires M2c polarization and MerTK induction. J Immunol (2012) 189:3508-20. doi: 10.4049/jimmunol.1200662

23. Zagorska A, Traves PG, Lew ED, Dransfield I, Lemke G. Diversification of TAM receptor tyrosine kinase function. Nat Immunol (2014) 15:920-8. doi: 10.1038/ni.2986

24. Jakubzick CV, Randolph GJ, Henson PM. Monocyte differentiation and antigen-presenting functions. Nat Rev Immunol (2017) 17:349-62. doi: 10.1038/nri.2017.28

25. Fleuren ED, Hillebrandt-Roeffen MH, Flucke UE, Te Loo DM, Boerman OC van der Graaf WT, et al. The role of AXL and the in vitro activity of the receptor tyrosine kinase inhibitor BGB324 in Ewing sarcoma. Oncotarget (2014) 5:12753-68. doi: 10.18632/oncotarget.2648

26. Dantas-Barbosa C, Lesluyes T, Loarer FL, Chibon F, Treilleux I, Coindre JM, et al. Expression and role of TYRO3 and AXL as potential therapeutical targets in leiomyosarcoma. Br J Cancer (2017) 117:1787-97. doi: 10.1038/bjc.2017.354

27. Potratz J, Tillmanns A, Berning P, Korsching E, Schäfer C, Lechtape B, et al. Receptor tyrosine kinase gene expression profiles of Ewing sarcomas reveal ROR1 as a potential therapeutic target in metastatic disease. Mol Oncol (2016) 10:677-92. doi: 10.1016/j.molonc.2015.12.009

28. Schoumacher M, Burbridge M. Key Roles of AXL and MER Receptor Tyrosine Kinases in Resistance to Multiple Anticancer Therapies. Curr Oncol Rep (2017) 19:19. doi: 10.1007/s11912-017-0579-4

29. Verma A, Warner SL, Vankayalapati H, Bearss DJ, Sharma S. Targeting Axl and Mer kinases in cancer. Mol Cancer Ther (2011) 10:1763-73. doi: 10.1158/ 1535-7163.MCT-11-0116 
30. Brand TM, Iida M, Stein AP, Corrigan KL, Braverman CM, Luthar N, et al. AXL mediates resistance to cetuximab therapy. Cancer Res (2014) 74:5152-64. doi: 10.1158/0008-5472.CAN-14-0294

31. Zhang Z, Lee JC, Lin L, Olivas V, Au V, LaFramboise T, et al. Activation of the AXL kinase causes resistance to EGFR-targeted therapy in lung cancer. Nat Genet (2012) 44:852-60. doi: 10.1038/ng.2330

32. McDaniel NK, Cummings CT, Iida M, Hulse J, Pearson HE, Vasileiadi E, et al. MERTK Mediates Intrinsic and Adaptive Resistance to AXL-targeting Agents. Mol Cancer Ther (2018) 17:2297-308. doi: 10.1158/1535-7163.MCT-17-1239

33. Yokoyama Y, Lew ED, Seelige R, Tindall EA, Walsh C, Fagan PC, et al. Immuno-oncological Efficacy of RXDX-106, a Novel TAM (TYRO3, AXL, MER) Family Small-Molecule Kinase Inhibitor. Cancer Res (2019) 79:19962008. doi: 10.1158/0008-5472.CAN-18-2022

34. Werfel TA, Elion DL, Rahman B, Hicks DJ, Sanchez V, Gonzales-Ericsson PI, et al. Treatment-Induced Tumor Cell Apoptosis and Secondary Necrosis Drive Tumor Progression in the Residual Tumor Microenvironment through MerTK and IDO1. Cancer Res (2019) 79:171-82. doi: 10.1158/0008-5472.CAN-18-1106

35. Stanford JC, Young C, Hicks D, Owens P, Williams A, Vaught DB, et al. Efferocytosis produces a prometastatic landscape during postpartum mammary gland involution. J Clin Invest (2014) 124:4737-52. doi: 10.1172/ JCI76375

36. Chen Y, Favata M, Pusey M, Li J, Lo Y, Ye M, et al. Identification of pAKT as a pharmacodynamic marker for MER kinase in human melanoma G361 cells. Biomark Res (2020) 8:4. doi: 10.1186/s40364-020-0184-9

Conflict of Interest: JR-D, MF, KLa, PF, YL, GY, CS, XWa, SSe, KK, JH, MY, SSp, $\mathrm{XWe}, \mathrm{CH}, \mathrm{WY}, \mathrm{MC}$, and $\mathrm{HK}$ are employees of and own stock in Incyte Corporation. KLi, RW, Y-LL, and PS are former employees of and may own stock in Incyte Corporation.

Copyright (c) 2020 Rios-Doria, Favata, Lasky, Feldman, Lo, Yang, Stevens, Wen, Sehra, Katiyar, Liu, Wynn, Harris, Ye, Spitz, Wang, He, Li, Yao, Covington, Scherle and Koblish. This is an open-access article distributed under the terms of the Creative Commons Attribution License (CC BY). The use, distribution or reproduction in other forums is permitted, provided the original author(s) and the copyright owner(s) are credited and that the original publication in this journal is cited, in accordance with accepted academic practice. No use, distribution or reproduction is permitted which does not comply with these terms. 